Entstehung secundärer Geschwulstknoten durch paradoxe Embolie und hätte ich densèlben, wie ich glaube, ohne weiteres meinen vor nicht langer Zeit mitgetheilten Fällen dieser Art von Geschwulstmetastase anreihen können ${ }^{1}$ ). Ich habe es nicht gethan, weil man den Einwurf machen konnte, es hätte möglicher Weise die Verschleppung von Geschwulstelementen durch Einbruch in die Aorta stattfinden können und weil man als Beweis hierfür den Milzinfarct anführen konnte, der ja jedenfalls doch auch embolischer Natur war und für den sich keine andere Quelle auffinden liess.

\title{
XI.
}

\section{Neuer Beitrag zur Sublimatintoxication nebst Bemerkungen über die Sublimatniere.}

\author{
Von Dr. Eduard Kaufmann, \\ Docenten und I. Assistenten am pathologischen Institut zu Breslau.
}

(Hierzu Taf. VIII.)

Nach Abschluss meiner Arbeit über die Sublimatintoxication $^{2}$ ) hatte ich Gelegenheit eine Section zu machen, welche mir gestattete, die in meiner Arbeit niedergelegten klinischen und pathologisch-anatomischen Daten noch einmal an einem typischen Falle zu controliren. Ich veröffentliche denselben um so lieber, als er mir zugleich den Anlass bietet, hier auf einen Punkt im pathologisch-anatomischen Bilde, nehmlich die Kalkniere, noch einmal näher einzugehen.

\section{Krankengeschichte.}

Am 9. Juli 1888 nahm die 20jährige Wärterin E. Petzoldt, angeblich in Entrüstung über einen Brief ihres Geliebten, gegen 7 Uhr Abends 200 bis $300 \mathrm{~g}$ einer 4 procentigen Lösung von Sublimat, also die enorme Quantitä von $8-12 \mathrm{~g}$ Sublimat, per os zu sich. Bald darauf trat

I) Dieses Archiv. 1889. Bd. 115. S. 71.

2) Die Sublimatintoxication. Beiträge zur Geschichte, Klinik und pathor logischen Anatomie derselben nebst experimentellen Untersuchungen zur Theorie ihres Wesens. Breslau. W. Koebner. 1888. 


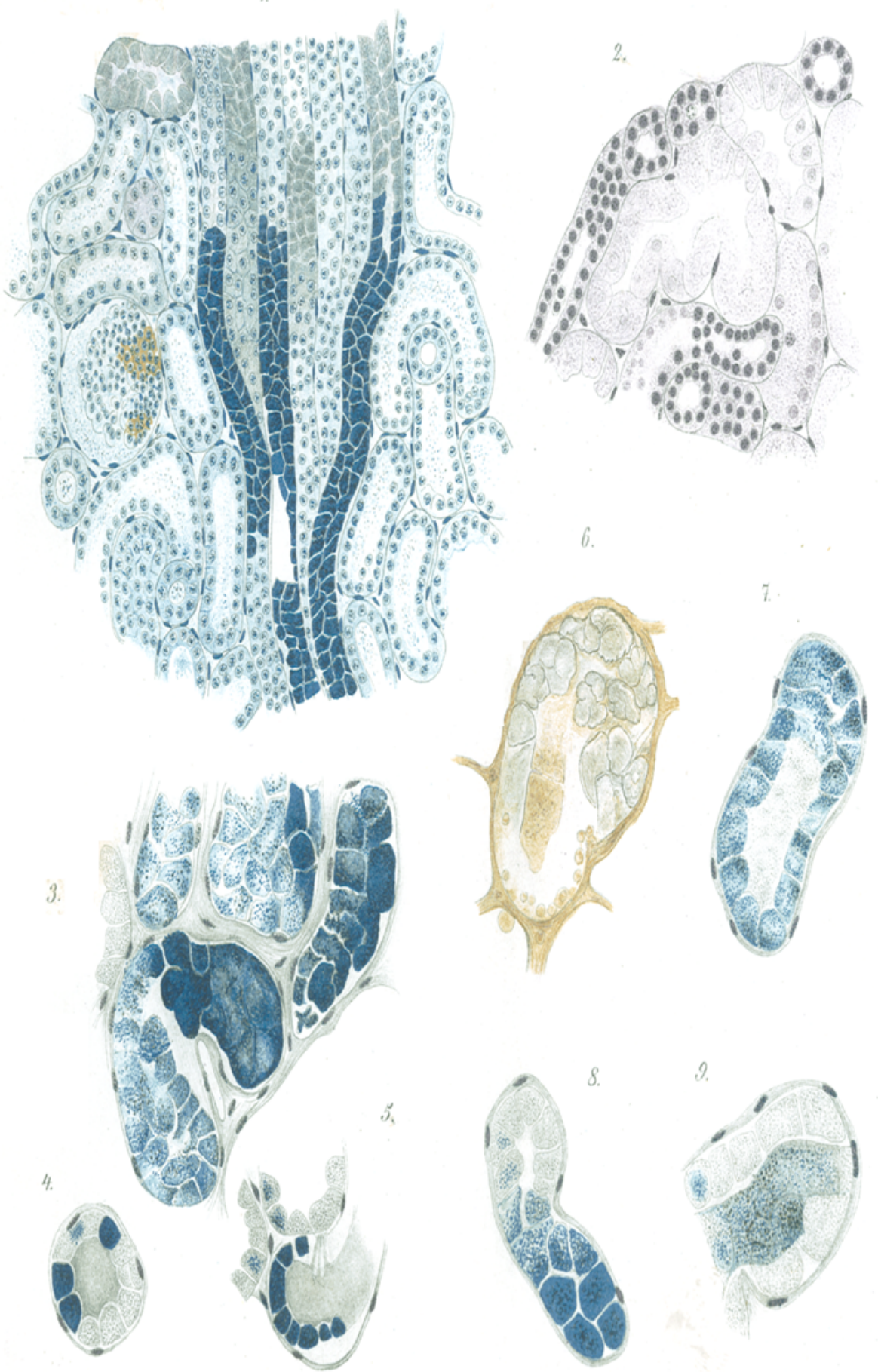


Erbrechen ein, welches die Nacht über anhielt. Das Erbrochene war dünnflüssig, blutig nnd entbielt grössere Flocken. Die Patientin klagte über Kraizen und Schmerzhaftigkeit im Halse und über starke Leibschmerzen. Das Gesicht war geröthet, die Augen thränten. Am rechten Auge wurde eine subconjunctivale Blutung constatirt.

Am 1. Tage der Bebandlung bekam Patientin das Eiweiss von 10 Eiern, was sie jedoch sofort erbrach. Ausserdem bestand die Medication in der stündlichen Darreichung von einem Esslöffe] 0,5procentiger Argentum nitricum-Lösung. Das Sensorium war nicht benommen; der Puls war klein und etwas beschleunigt. Es erfolgte an diesem Tage kein Stuhl, dagegen wurde Urin gelassen.

Am folgenden Tage (11. Juli) war der Puls besser, voll, weich und nicht beschleunigt. Die Schmerzhaftigkeit im Leibe hatte etwas nachgelassen, dagegen bestanden die Schmerzen im Halse fort. Nunmebr erfolgte auch ein dünnfï̈ssiger, braungrüner, äusserst übelriechender Stuhl. Die Urinsecretion zeigte bereits eine schwere Alteration; der Harn war spärlicb, enthielt viel Eiweiss und granulirte Cylinder. Die Ordination bestand in Emulsio amygd. mit Mucilago Salep, und Stärkeklystieren mit Opium. Am Abend erbielt die Kranke $2 \mathrm{~g}$ Sulfonal und schlief die Nacht ziemlich gut.

Am 4. Tage trat Anurie ein, welche auch die folgenden Tage fortbestand. Der Stuhl war frei von Blut. Das Sensorium war etwas benommen und Patientin war sehr schläfrig. Es erfolgten prophylactische Ausspülungen des Mundes mit Kali chloricum. Trotzdem stellte sich

am 5. Tage eine starke Stomatitis und Salivation. ein. Der Puls war gut, voll und weich. Verordnet wurde gegen die Anurie Infusum fol. digitalis mit Kali aceticum.

An den folgenden Tagen wurde wieder spärlich Urin gelassen und am dritifolgenden sogar eine grössere Menge. Der Stubl enthielt nur wenig Blut. Gegen die immer heftiger werdende Stomatitis wurden Pinselungen mit Acidum hydrobromicum angewandt. Weiterhin kamen beisse Bäder, heisse Einwicklungen und Stärkeklystiere mit Opium in Anwendnng. Trotz der Behandlung veränderte sich der Zustand nicht zum Besseren. Die Urinmenge blieb andauernd anf ein Minimum reducirt, mässige Diarrböen bestanden fort und die Stomatitis machte immer grössere Fortschritte.

Am 15. Krankheitstag trat eine bedeutende Verschlimmerung ein. Während sich nebmlich die Temperatur bisher annähernd normal verhalten batte, sank dieselbe an diesem Tage auf 35,1 herab, um sich nicht mebr über 35,6 zu erheben. Dazu trat ein zweites, neues Symptom in der Gestalt sebr starker Dyspnoe, mit lebhaftem Angstgefühl. Ausserdem trat Erbrechen ein, das scbliesslich nur noch die Aufnabme von wenig Wasser und Wein gestattete. Die Diarrhöen waren wenig profus und obne blutige Beimengungen.

In den 4 folgenden Tagen nahm die Schwäche irnmer mehr zu und das Erbrechen steigerte sich bis zur Unstillbarkeit. Am 18. Krankheitstag war das Sensorium benommen, die Herzaction sehr schwach, die Athmung wurde iminer beschwerlicher und ganz unregelmässig; am Tage vor dem Tode traten 
Convulsionen ein und unter Zunahme der Symptome von Seiten des Herzens und der Athmung trat

am 19. Krankheitstage, Mittags $1 \frac{1}{4}$ Uhr der Tod ein.

Wenn wir die Daten der vorgeführten Krankengeschichte mit den anderweits bekannten Fällen zusammenhalten, so ist zunächst hervorzuheben, dass trotz der enorm grossen Dosis der Tod sehr spät"eintrat. Durch 19 Tage zog sich die Vergiftung hin, trotzdem 8-12 g Sublimat genommen wurden. Es findet dieses Verhalten keine Analogie in den bekannten Fällen. Im Allgemeinen zwar sind die Vergiftungen per os die am wenigsten folgenschweren und zwar offenbar deshalb, weil alsbald nach der Einverleibung Erbrechen eintritt, so dass zur Resorption der ganzen Dosis gar keine Zeit gelassen wird. Viel schlimmer steht es mit den Fällen, in denen das Gift von der Vagina, dem Uterus, dem Peritonäum, von einer grossen Wundfläche, subcutan oder per rectum ${ }^{1}$ ) aufgenommen wird. Hier genügen oft nur kleine Mengen, um den Tod herbeizuführen. Diese Verhältnisse finden sich in meiner Schrift über die Sublimatintoxication tabellarisch zusammengestellt. Wie sehr aber auch bei den Vergiftungen einer Art die individuellen Versehiedenheiten zur Geltung kommen, das zeigt der Vergleich nur weniger Fälle. So nahm in dem Falle von Szabó eine 40 jährige Wärterin $5 \mathrm{~g}$ Sublimat in $300 \mathrm{~g}$ Kaffee $\mathrm{zu}$ sich; trotzdem sofort die Magenpumpe in Anwendung kam, verstarb die Person bereits nach 68 Stunden. In dem Falle, den Armstrong berichtet, trank ein 45 jähriger trunksüchtiger Krankenwärter $6 \mathrm{~g}$ Sublimat in $60 \mathrm{ccm}$ absolutem Alkohol und genas nach $11 \mathrm{Ta}$ gen wieder. Diesem Menschen kam wahrscheinlich seine chro-

1) Fall von Huber (Zeitschr. f. klin. Med. Bd.XIV. 5. u. 6. Heft. 1888); es handelte sich bier um einen irrtbümlich mit Sublimatlösung anstatt mit essigsaurer Thonerde vorgenommenen Einlauf, der $0,75 \mathrm{~g}$ Sublimat enthielt. Alsbald trat Erbrechen und reichliche Darmentleerung ein und am 5. Tage erfolgte der Tod unter Collapserscheinungen. Bei der Section fanden sich stellenweise starke Injection und Nekrose des Darms; die Nieren boten keine Verkalkungen. Es finden sich nur. 2 andere Fälle in der Literatur, in denen der Darm die Eingangspforte für das Gift war. (Hofmann, Lehrb. Il. Aufl. S. 548 und Bokelmann, Centralblatt von Fritsch 1884. No, 11, S. 61 ; letctere Vergiftung ging in Genesung über. 
nische Gastritis zu Statten, in dem einmal die Resorption beeinträchtigt war und das andere mal sofort Verbindungen von Sublimat mit den reichlichen Schleimmassen eingegangen wurden. In dem Falle von Barthélmy dagegen ging das jugendliche Individuum nach nur $0,7-0,8 \mathrm{~g}$ Sublimat in alkoholischer Lösung nach 9 Tagen zu Grunde.

Was die Symptome des Krankheitsbildes angeht, so haben wir in dem Würgen und Erbrechen, der Schmerzhaftigkeit im Schlund und in der Magengegend bekannte Anzeichen der $\mathrm{Hg}$ Vergiftung. Auffallend ist jedoch in unserem Fall, dass die Diarrhöen erst am 3. Tage begannen; in anderen Fällen erfolgten dieselben bedeutend früher, zuweilen schon nach wenigen Stunden. Auch im Verlauf der Krankbeit zeigten die IDiarrhöen kein der Grösse der Vergiftungsdosis entsprechendes Verhältniss. Andere Fälle boten fast unausgesetzte, blutige Stühle und in ganz schweren Fällen gingen mit dem blutigen Koth grosse Schleimhautfetzen ab. In dem vorliegenden Fall waren die Stühle wenig profus und nur vorübergehend blutig.

Das Verhalten des Urins dagegen, der hohe Gehalt an Albumen, die starke Verminderung bis zu fast völliger Anurie, schliesst sich demjenigen der bekannten Fälle an. Auch die Temperaturverhältnisse sind charakteristisch. Wie erwähnt bestanden in den letzten Lebenstagen subnormale Temperaturen. In anderen Fällen erreichten dieselben jedoch einen weit niedrigeren Grad, so 34,4 im Fall Levy und sogar 33,4 am 2.Tage vor dem Tode im Falle von Loewy.

Die nervösen Erscheinungen, welche sonst bisweilen beobachtet wurden, sind in unserem Fall reichlich vorhanden. Angstgefühl, starke Aufregung, Schlaflosigkeit und auf der anderen Seite völlige Somnolenz und Bewusstlosigkeit wurden verzeichnet. Etwas Charakteristisches sind dergleichen Symptome nicht für die Sublimatvergiftung; berichtet doch Keller ïber einen Fall von Vergiftung, die sich an eine Totalexstirpation des Uterus anschloss und in 78 Stunden lethal endigte, bei welchem während des ganzen Verlaufs völlige Euphorie bestand, und im tödtlichen Falle von Netzel bestand anfangs Somnolenz, die sich nachher wieder verlor.

Sehr charakteristisch gestaltete sich nun der Befund der am folgenden Tag von mir ausgeführten Autopsie. 
Was zunächst das Berz angeht, so war dasselbe in seinem rechten Antheil weit, dabei jedoch so schlaff in der Wandung des Ventrikels, dass derselbe wie ein angestocbener Gummiball zusammengefallen erschien. Der linke Ventrikel füblte sich ziemlich fest an, seine Wanddicke betrug durchweg $1,5 \mathrm{~cm}$; der linke Ventrikel war leer, während der rechte wenig dünnes Blut enthielt. Farbe der Musculatur des rechten Herzens hellgraubraun, links rothgrau. Unter dem Endocard am Conus pulmonalis eine fast erbsengrosse Blutung.

Linke Lunge auf der Oberfäche ausserordentlich scheckig, besonders hinten. Auf der Scbuittfläche wechseln besonders im Unterlappen hellere und dunklere Partien mit einander ab. Die dunkleren haben einen schwarzrothen Farbenton und es lässt sich blutige Flüssigkeit von ihnen abstrejchen. Auf der Scbnittfäche der recbten Lunge berrscht dasselbe auffallende Verhältniss. Unter der Pleura dieser Lunge finden sich besonders hinten und unten grössere Blutungen bis zu 5-Pfennigstückgrösse. Die Bronchialschleimhaut dunkelroth, mit schleimigen zähen Massen bedeckt.

Die Schleimhaut des Pharynx is dunkelroth gefärbt. Auffallend ist ein bellerer, ungefähr fingerbreiter Streifen, welcher quer über die Pharynxschleimhaut wegzieht und in der Höhe des oberen Randes der Epiglottis liegt. Nach unten setzt sich die Röthung bis zur Höbe des Aryknorpels fort. Am Oesophagus ist bemerkenswerth, dass gleich unterhalb der Möbe des Keblkopfes in einer Ausdebnung von $8 \mathrm{~cm}$ eine hellere grauweisse Färbung anftritt, welche durch Strecken von verdickten Epithelien bedingt wird, welche streifenförmig von oben nach unten verlaufen und sich ein wenig über die vascularisirte, leicht geröthete Ungebung erheben. Weiter unten ist der Oesophagus glatt, ödematös.

Der Magen ist mit einem grünen Inhalt gefült. Die Falten sind sebr starlz ausgeprägt, sulzig ödematös. Hier und da bemerkt man auf der Höhe der Falten kleine Blutungen. Nach dem Pylorus zu wird die Schleimbaut blasser; um so deutlicher heben sich fleckweise submucöse, stark injicirte Venen ab. Besonders anffallend ist eine Veränderung, die sich in der Magenschleimbaut da findet, wo dieselbe stark sulzig ödematös und verfärbt ist, also vorzüglich auf den stark ausgeprägten Falten und dann in der Ausdehnung eines kleinen Handtellers nahe der Cardia, ferner im Duodenum, welches im Uebrigen auf der Höhe einzelner Falten von grünbraunem Farbenton und glasig-sulziger Beschaffenheit ist. Es finden sich an den bezeichneten Stellen kleinste weiss-gelbliche sprenkelchen, die ausserordentlich zahlreich und dicht bei einander oben in der Schleimbaut liegen, und welche man für Auflagerungen halten lönnte; sie lassen sichjedoch nicbt abspälen. Die frisch vorgenommene Untersuchung mikroskopischer Scheerenschnitte und $Z$ upfpräparate ergab, dass es sich um mineralische Einlagerungen in der Epithelschicht der Schleimhaut hadelte; sie bildeten Klümpchen und drusenförmige Bröckel, welche sich auf Zusatz von Salzsäurelösang unter Koblensäureentwicke. 
lung lösten und nach Zusatz von $25 \mathrm{pCt}$. Schwefelsäure Gypskrystalle hinterliessen; es war also Kalk. Bei der Auflösung einzeln berausgezupfter Krämel konnte man unter dem Mikroskop sehr oft constatiren, dass zellenartige Gebilde nach der Entkalkung restirten.

Im Jejun am sind zahlreiche Falten von grünbraunem Farbenton und glasiger Beschaffenbeit. Im Tleum sehr lebhaft venöse Hyperämie und zahlreiche kleinste Schleimbantblutungen. Im Dickdarm keine Veränderungen von Bedeutung; er ist blass und zeigt nur an ein par Stellen auf der. Höhe der Falten kleine Blutungen.

Nachdem der Dickdarm abpräparirt war, wurde der Füllungszustand der abdominalen Gefässe constatirt. Hierbei zeigte sich eine sebr starke Füllung im venösen System; die Vena cava war stark erweitert und batte das Aussehen einer dicken Wurst; in ihrer Nachbarschaft befanden sich geschwollene dunkel grauroth gefärbte Lymphdrüsen.

Milz, 10 lang, 6 breit, $3 \frac{3}{2}$ dick. Consistenz ziemlich fest. Trabekel und Follikel sehr deutlich. Farbe der Pulpa bräunlichroth.

Leber. Auf der glatten Oberfäche heben sich viele blassere Bezirke von gelblichgrauer Farbe ab. Auf der Schnittfläche wechseln hellere und dunklere, gelblichgraue und rotbbräunliche Partien mit einander ab.

Gallenblase sehr stark gefüllt. Galle sebr dünnflüssig, von rothbräunlicher Farbe, welche in's Grüne spielt. 'Pankreas nicht verändert.

Die Hirnsinus stark gefüllt mit flüssigem Blut. Rinde des Gehirns blass; auf den Schnittfächen der Marksubstanz erscheinen zahlreiche Blutpunkte, aus denen fiüssiges Blut bervorquillt.

Beschreibung der Nieren folgt später.

Sehr auffallend sind an diesem Befund die geringen Veränderungen im Darm, das Fehlen nekrotischer Veränderungen vor Allem im Dickdarm. Letzterer zeigte nicht einmal Röthung und war durchweg frei von jeder schwereren Veränderung. Dieser Befund ist bei der Sublimatvergiftung nicht neu und er zeigte sich z. B. in dem von Scheuthauer secirten Fall von Szabó. Auch ein Fall von Maschka gehört hierher. Während diese Fälle jedoch ziemlich rasch tödtlich endeten, ist in unserem Falle der Mangel fast jeder Veränderung im Dickdarm bei der 19 tägigen Krankheitsdauer immer noch sehr auffallend. Jedenfalls spricht dieser Fall wieder dafür, dass, wie ich früher ${ }^{1}$ ) entschieden betonte, von einer Aetzwirkung des Sublimats im Darm, welche u. A. Liobreich annahm und was. sich wie eine ewige Krankheit bis in die

1) a. a. $0, \mathrm{~S}, 112$ u. ff. 
neuesten pharmakologischen Lehrbücher forterbt, gar keine Rede sein kann, wie das auch neuerdings Virchow') ausdrücklich hervorhob. Wie die leichteren und schweren Veränderungen, die sog. Diphtherie des Darms zu Stande kommen, warum die Veränderungen in dem einen Fall fehlen, in dem anderen stark entwickelt sein können, das ist nach meinen Untersuchungen nicht schwierig zu erklären. Es sei mir daher gestattet, in nuce meine Auffassungen von dem Wesen der Sublimatvergiftung zu exponiren. Das Wesen der Sublimatintoxication beruht auf einer Alteration des Blutes durch das Gift. Grosse Dosen bewirken, wie wir in zahlreichen Thierversuchen sahen, direct tödliche Gerinnung im Herzen und den grossen Gefässen, in den anderen Fällen lassen sich Verstopfungen in ausgedehnten $\mathrm{Ca}$ pillarbezirken nachweisen und zwar durch die Autoinfusion der Organe des Thieres mit färbenden Flüssigkeiten nach dem Vorgang von Silbermann. Während ich in meiner früheren Arbeit nur Indigcarminlösung anwandte, habe ich nachher mit Eosin-Kochsalzlösung (Silbermann) noch brauchbare Resultate erhalten. Vor allem weisen hierbei die Lungen einen grossen Ausfall von Capillarbezirken auf, Verstopfungen, gegen welche das Herz bis zur endlichen Insufficienz arbeitet. Daher die venöse Hyp̀erämie und die arterielle Leere. Mikroskopișch constatirt man, dass die Capillaren in den ungefürbten Bezirken so vollgepfropft sind mit rothen Blutkörperchen, dass dieselben unter Verschwinden der $Z$ wischenflüssigkeit fest gegeneinander gepresst sind und dass ferner in vielen Capillaren eine totale Homogenisirung der Blutkörperchen und die Umwandlung in einen glasigen Ausguss von rother Farbe stattgefunden hat, welcher sich auf Kosten der Sonderexistenzen der Blutkörperchen bildete; es handelt sich also um Stase und Bildung von rothen Thromben in den Capillaren. Schwierig und noch nicht gelöst ist die Frage über die Natur der die Thrombosen bewirkenden Momente, ob diese in der Schädigung der rothen Blutkörperchen, welche wir mikroskopisch constatirten, zu suchen sind, oder ob Fibrinferment auftritt, wofür eine Versuchsreihe in unseren Thierexperimenten spricht, wodurch Gerinnungen des Blates und

3) Sitz. der berl. med. Gesellsch, 21, Nov. 18s8, 
mittelst derselben Pfröpfe in den kleinsten Gefässen sich herstellen, welche dann Stase im Gefolge haben. Beide Momente spielen nach unserer Meinung mit. Wie dem aber auch sei, die Hauptsache ist, dass die Verlegungen der Gefässbahnen nachweislich bestehen.

Ausser in den Lungen findet man ungefärbte Bezirke in der Leber, dem Magen, den Nieren und dann oft in bervorragender Weise im Darm. Die Behinderung der Circulation in den Car pillaren, in welchen das Blut festsitzt, veranlasst den bekrotischen Zerfall der betreffenden Abschnitte der Schleimhaut, welcher durch diapedetische Blutung aus den stasirten Capillarbezirken begünstigt wird. Dass grade die Höhen der Falten zuerst und oft allein von den Veränderungen betroffen werden, hängt damit zusammen, dass durch das sich Gegeneinanderpressen dieser Stellen bei den krampfhaften Contractionen des Darmrohres eine Erschwerung des Stromes durch die Capillaren stattfindet; dass Verstopfungen in den Capillaren zu bilden, dieser Umstand bei der Tendenz des Sublimatblutes der Stockung der Circulation günstig ist, ist zwanglos anzunehmen. In den durch die geschilderten Circulationsstörungen ihrer Widerstandsfähigkeit und weiterhin ihres Lebens beraubten Schleimhautabschnitten siedeln sich im Darm, besonders im Dickdarm stets reichlich vorhandene Bakterien an, welche eine secundäre Entzündung anregen, welche zuweilen den Charakter einer schweren Dysenterie annehmen kann. Nach der vorhin gegebenen Erklärung der Sublimatwirkung als der eines Blutgiftes, welches Thrombose und Stase bewirkt, wird es verständlich, warum eventuell die schweren Darmveränderungen fehlen können; es haben sich dann aus Gründen, welche man freilich im einzelnen Fall - wie in dem oben berichteten - nicht anzugeben vermag, keine Capillarverstopfungen dort gebildet und es fehlen natürlich auch die in Nekrose bestehenden Folgeerscheinungen. Wären die Darmveränderungen durch eine locale Aetzwirkung bedingt, so würde dieser Befund unverständlich sein, denn warum sollte der Hauptexcretionsort des $\mathrm{Hg}^{1}$ ), der Darm, diesmal von der Aetzwirkung verschont geblieben sein?

1) Quantitative Bestimmungen über die Ausscheidung des $\mathrm{Hg}$ durch die Galle, welcher der Darm zum guten Theil seinen Hg-Gehalt verdankt, 
Durch die Hämorrhagien im Darm, den Untergang rother Blutkörperchen, sowie vorzüglich durch die Behinderung des kleinen Kreislaufes wird eine bedeutende arterielle Anämie im gesammten Organismus gesetzt.

Was nun die Nieren angeht, so stimmt zunäehst die makroskopische Beschreibung mit dem überein, was ich früher (l. c.) auseinander gesetzt habe. Im Protocoll ist darüber Folgendes verzeichnet: Linke Niere auffallend dick und weich; $12 \mathrm{~cm}$ lang, 5 breit, 4,5 dick. Die Rinde ist von aussen glatt, die Venensterne sind stark injicirt und heben sich scharf aus der grauweissen Oberfläche ab. Sodann bemerkt man auf der Oberfläche kreideartige Pünktchen von unregelmässiger Gestalt und sehr geringer Grösse. Auf der Schnittfläche erscheint die Rinde sehr breit, gelbgrauweiss gefärbt; sie ist eigenthümlich schraffirt, indem rothe und gelblichgrauweisse Streifen mit einander abwechseln. Ferner ist die Schnittfläche der Rinde mit sehr zahlreichen, kreideartigen Stippchen von sehr unregelmässiger Gestalt gesprenkelt, die oft eine gewisse Anordnung wie gewundene Harnkanälchen erkennen lassen. Glomeruli sind nicht zu sehen; auch sonst ist die normale Zeichnung nicht zu erkennen. Die Marksubstanz ist scharf gegen die Rinde abgesetzt, von schöner, dunkelrother Farbe. In derselben ist auch eine Streifung $\mathrm{zu}$ bemerken, indem rothe und rothgrane Jängsstriche abwechseln. Rechte Niere: kleiner, blasser wie die linke; wesentlich von heller grangelbweisser Farbe, ohne die Schraffirung, welche auf der Schnittfiäche der linken Niere sichtbar ist. Sonst das gleiche Verhalten wie bei der anderen Niere.

Den Umstand, dass kreideartige Absätze makroskopisch von aussen und auf der Schnittfläche der Rinde so dentlich sichtbar waren, betone ich ausdrücklich, weil das in anderen Beobachtungen vielleicht nicht immer der Fall ist; wenigstens konnten, wie Virchow ${ }^{1}$ ) angiebt, in dem von ihm berichteten Fall von Cyanquecksilbervergiftung die Kalksalze makroskopisch absolut nicht constatirt werden, trotzdem sich die Nieren mikroskopisch

finden sich in der Dissertation von Hassenstein, Versuche über Quecksilberansscheidung durch die Galle, Königsberg 1879, welche unter Naunyn verfasst wurde.

) a. a. 0 , 
stärker wie je mit Kalkconcretionen erfüllt zeigten. In unserèm neuen Fall bot die makroskopische Diagnose gar keine Schwierigkeiten, sodass man die Kalkkrümelchen ohne weiteres zum Zweck der mikrochemischen Untersuchung herauszupfen konnte. Auch in den früher von mir beschriebenen Fällen war die Verkalkung leicht zu sehen. Ausser in dem erwähnten Falle von Virchow wurde die Verkalkung makroskopisch auch nicht notirt, z. B. in dem Falle von Steffek, wo erst mikroskopisch, die freilich geringe Verkalkung sich herausstellte ${ }^{1}$ ).

So sehr die Niere makroskopisch einer grossen weissen Niere ähnlich sieht, also einer parenchymatösen Entzündung, so different stellt sich der mikroskopische Befund im Vergleich mit einer ächten parenchymatösen Nephritis dar, ein Unterschied, auf den schon Prevost aufmerksam machte. Es handelt sich nicht sowohl um eine entzündliche Degeneration; als um eine Nekrose der Epithelien auf nicht entzündlicher Basis. Um dies im vollen Umfang constatiren zu können, muss man einen so reinen Fall vor sich haben, wie es der unserige ist, wo im Gegensatz zu der Mehrzahl der Fälle - auch zu den 2 früher von uns mitgetheilten - infectiöse und andere Schädigungen, welche eine Entzündung der Niere hätten veranlassen können, mit Sicherheit auszuschliessen sind. Man ist überrascht, bei der frischen Untersuchung keine Spur von fettiger Degeneration der Epithelien zu finden, wozu der makroskopische Anblick verleiten könnte. Es lassen sich nun folgende Veränderungen der Epithelien der Harnkanälchen aus dem mikroskopischen Bilde ableiten. Die Epithelien worden körnig, quellen auf und ihre Kerne werden nicht mehr sichtbar; die normalen Contouren verschwinden mehr und mehr und unförmige, plumpe, oft vieleckige Zellen finden wir anstatt des regelmässigen Epithelsaumes. Diese Veränderungen finden sich sowohl beim Menschen, als auch beim Kaninchen. In Fig. 1 sehen wir diese nekrotischen Epithelien, welche bei der Hämatoxylinfärbung einen schmutzig gramen Farbenton angenommen haben und unregelmässige Gestaltung zeigen; Kerne sind in ihnen nicht gefürbt. Prevost, Senger und Huber bezeichnen die Veränderung gleichfalls als Nekrose. Von dieser

1) Es empfiehlt sich daher gewiss, wie das auch Virchow räth, in allen verdächtigen Fällen eine mikroskopische Untersuchung vorzunehmen. 
Veränderung sind beim Mensehen vorzugsweise die gewundenen Kanälehen, bei Kaninchen vorzüglich die Tubuli recti der Rinde, jedoch auch gewundene Harnkanälchen betroffen (s. Fig. 1). Neben den nekrotischen Epithelien sind andere zu sehen, welche nur eine leichte Trübung zeigen und wieder andere Abschnitte zeigen ein normales Verhalten. Die Sublimatniere beim Menschen ebenso wie die beim Kaninchen zeigt, wie ich das betonen muss, ein sprungweises oder heerdweises Auftreten der degenerativen Veränderungen, es handelt sich absolut nicht um einen von Haus aus gleichmässig über die ganze Niere ausgebreiteten Prozess. Dieser Befund, der schon von Saikowsky für das Kaninchen angegeben wurde, ist auch von Huber für die menschliche Niere angegeben worden. Die Betrachtung unserer Fig. 1 einer Sublimatniere des Kaninchens zeigt dies Verhältniss auf das Deutlichste. Die Ausbreitung der degenerativen Veränderungen der Epithelien ist offenbar in den verschiedenen Fällen sehr verschieden und es kann schliesslich die Veränderung so stark werden, dass von gesunden Tubuli contorti fast gar nichts mehr zu sehen ist. Dies ist auch in unserem neuen Falle von 19 tägiger Dauer eingetreten und unter diesen Umständen ist von einem heerdweisen Auftreten der Veränderungen kaum noch etwas zu sehen, was natürlich nicht im geringsten gegen eine Zusammensetzung aus ursprünglich getrennten Heerden spricht. In den Präparaten dieser Nieren sind die Epithelien der gewundenen Kanälchen fast sammt und sonders mehr oder weniger verändert, theils getrübt, theils nicht tingirbar in den Kernen; an frischen und gehärteten Präparaten fehlt fast überall der regelmässige Epithelbesatz im Bereich der gewundenen Harnkanälchen; abgesehen von Gestaltsveränderungen normal sitzender Epithelien sind dieselben vielfach von der Basalmembran abgelöst und beliebig im Lumen gelegen; auffallend ist ihre Vergrösserung zu voluminösen, oft mit mehreren Ecken versehenen Zellen; andere sind in kleinere Stüeke auseinander gebröckelt, wieder andere sind zusammengebacken und theilweise in feinkörnige oder hyaline Cylinder umgewandelt.

In dem beschriebenen Zustand - der Verkalkungen wird später gedacht werden - sehen wir die kalklose Sublimatniere im höchsten Grade der Veränderungen. Diese Verbält- 
nisse zeigt Fig. 2 nach dem Alkoholpräparat einer Sublimatniere gezeichnet, welche keine Verkalkungen bot und welche einem im Juni 1887 zur Beobachtung gelangten Falle entstammt. Ausserdem fand sich in diesem Falle fettige Degeneration von Epithelien, welche in Abhängigkeit gebracht wurde von einer durch die bestehende hochgradige Sepsis bedingten parenchymatösen Degeneration ${ }^{1}$ ).

Wie kommt nun die Nekrose der Epithelien zu Stande? Offenbar handelt es sich dem histologisehen Charakter nach um den Process, den wir als Coagulationsuekrose oder anämische Nekrose zu bezeichnen gewohnt sind. Durch Anämie warde hier der Zelltod veranlasst. Dieselbe wirkt in verschiedener Weise schädigend auf die Niere ein. Zunächst besteht bei der Sublimatintoxication hochgradigste arterielle Anämie, gegen welche die Epithelien ausserordentlich empfindlich sind; auf der anderen Seite herrscht enorme venöse Hyperämie. Hierdurch erklären sich zunächst die Oligurie, das Auftreten des Albumens im Harn, sowie der eventuelle Vebergang körperlicher Elemente des Blutes in den Harn; die Eiweissgerinnungen finden sich nicht nur in den Harnkanälchen, sondern auch in den Glomeruluskapseln, ohne dass jedoch eine Alteration des Epithels der Schlingen nachzuweisen wäre. Auf der anderen Seite genügt dieses Missverhältniss in der Blutvertheilung bei längẹrer Dauer schon ausreichend, um die Vitalität der Epithelzellen der gewundenen Kanäle ernstlich zu gefährden. Dann zeigen sich aber weiterhin bei den Autoinfusionsversuchen mit gefürbten Flüssigkeiten bei Kaninchen fleckweise ungefärbte Gefässbezirke. An frischen Präparaten besonders deutlich ist ein Vollgepfropftsein in den Capillaren dieser Bezirke, wie wir es bei der Lunge, freilich viel ausgedehnter sehen, und zuweilen eine Homogeni-

1) Sobald eine erbeblichere fettige Degeneration der Epithelien eingetreten ist, findet, wie ich früher bereits betonte (a. a. O. S. 120), keine Verkalkung derselben statt. Diese Ansicht wird durch obigen Fall wiederum bestätigt. In der Dissertation von Weinbold, wo der Fall sich findet, ist kurz die Angabe gemacht, es haben sich die für Sublimatvergiftung charakteristischen Veränderungen in den Nieren gefunden; Kalkablagerungen waren jedoch, wie aus meiner mikroskopischen Untersuchung hervorgebt, nicht vorhanden. 
sirung der Blutsäule; eine rothe 'Thrombose, zu constatiren. Auch Blutaustritt, wohl durch Diapedese bedingt, ist in der Umgebung dieser Bezirke fleckweise zu constatiren, worauf schon frühere Beobachter, so Heineke, hinwiesen. Diese beiden genannten Umstände addiren sich um die Herabsetzung der Ernährung der betreffenden Nierenabschnitte zu bewirken. Vor allem die Epithelien der gewurdenen Kanälchen antworten auf diese Schädigung mit Nekrose, Einer directen Einwirkung des ausgeschiedenen Sublimates auf die Zellen eine Bedeutung für das Zustandekommen der Nekrose zuzuerkennen, bin ich nach meinen Untersuchungen nicht berechtigt; es spricht aber vor Allem ja das Moment dagegen, dass die graden Harnkanälchen beim Menschen wenig oder gar nicht betheiligt sind, was doch der Fall sein müsste, wenn das Quecksilber bei seiner Ausscheidung durch seinen Contact die Epithelien schädigte oder gar vernichtete.

Der Prozess der Capillarverstopfungen tritt heerdweise auf und hat aus Gründen, die ich früher ${ }^{1}$ ) auseinandersetzte, mit der hämorrhagischen Infarcirung gar nichts zu thun. Unmöglich konnte ich daher die Ansicht äussern, das eventuelle weitere Schicksal der absterbenden Epithelien - die Verkalkung - entwickle sich in derselten Weise wie bei einem alten hämorrhagischen Infarct, wie mir dies Virchow vorhält; nach Virchow's Auffassung soll ich in meiner Arbeit behaupten, dass beim Mercurialismus etwas Aehnliches geschehe, wie bei den hämorrhagischen Infarcten in der Niere selten beobachtet wird, wo innerhalb der infarcirten Theile eine Concretionsbildung vorkommt. Eives solchen Vergleiches bin ich mir gänzlich unbewusst.

Was nun die Kalkablagerungen in den Nieren angeht, so herrscht hierüber eine Verschiedenheit der Meinungen.

Virchow sagt, es handle sich um Kalkcylinder oder Krümel im Lumen der Kanäle gelegen, also an der Oberfläche der Epithelien; er setzt diesen Prozess in Analogie zu dem, was er als. Kalkmetastasen ${ }^{1}$ ) beschrieb, jene spontane Form von Kalkinfarct, welche dann auftritt, wenn durch

1) a. a. O. S. 116 .

2) Dieses Archiv Bd. s. 
Tumoren oder ulcerirende andere Prozesse des Scelets plötzlich viel Knochensubstanz eingeschmolzen und das Blat mit Kalksalzen überladen wird. Unter diesen Umständen sehen wir Verkalkungen in den Lungen, der Magenmucosa, der Gehirnarterien und auch in den Nieren eintreten. Ueber den Ort der Ablagerung der Kalksalze bei diesem Prozess in den Nieren könnte man in einigem Zweifel sein, wenigstens sind die Angaben, welche sich darüber finden, sehr spärlich und nicht ganz congruent. Was ich darüber in der literatur fand, sind folgende Passus: Virchow (s. Arch. Bd. 9 S. 620): „In den Nieren, welche gross, schlaff, sehr geschwellt, blass und besonders in der Rinde fettig degenerirt waren, zahlreiche verkalkte Harnkanälchen und Epithelien sowohl in der Rinde, als in den Pyramiden." Schläpfer (dieses Arch. Bd. VII. S. 160): "Unter allen mir bekannten Formen der Verkalkung entspricht diese hier am meisten der der papillären Harnkanälchen, die so oft vorkommt, und welche sich von einer einfachen körnigen Ablagerung auf die Tunica propria bis zu dicken krystallhellen und homogenen Massen verfolgen lässt." In den Lehrbüchern von Klebs, Perls, Birch-Hirschfeld wird die Kalkmetastase in den Nieren als Kalkinfaret in den graden Harnkanälchen der Pyramiden bezeichnet. v. Recklinghausen (Handbuch S. 174) spricht von einer Incrustation der Nierensubstanz mit Kalkkrümeln. Virchow (l. I. c.) bat nun neuerdings seine Auffassung noch einmal in nuce gegeben und hierans sind für unsere Betrachtungen besonders die Sätze wichtig, dass zunächst die Kalkablagerung bei der Kalkmetastase nichts zu thun haben soll mit dem gewöhnlichen Kalkinfarct, der wesentlich den $\mathrm{Pa}$ pillartheil der Marksubstanz einnimmt, sondern dass eine Kalkabsetzung in den Nieren genau auf dieselbe Weise wie bei der mercuriellen Form durch Concretionsbildung in den gewundenen Harnkanälchen sich vollziehe, indem der Kalk im Lumen, wie das Virchow für diese Niere annimmt, gelegen sei.

Die Ueberschwemmung des Blutes mit Kalk soll dadurch zu Stande kommen, dass, wie Prevost angab, eine Decalcinirung des Knochens unter dem Einfluss des Sublimates statt habe.

Meine Untersuchungen über diesen Punkt hatten mich nun zu einer anderen Auffassung geführt, welcher Virchow nicht 
beipflichtet. Ich behauptete, dass der Kalk, der stets in genügender Menge in den Gewebssäften und im Blut circulirt, sich in den Epithelien festsetzt, dort gebunden wird und zwar nachdem die Epithelien der gewundenen Kanälchen durch anämische Nekrose für die Kalkaufnahme tauglich gemacht worden sind. Die anämische Epithelnekrose, durch die oben erwähnten Momente bedingt, ist das Primäre, bald nachher erfolgt die Incrustation mit Kalksalzen. Hierzu ist die Annahme einer Decalcinirung der Knochen nicht erforderlich, wie die Versuche von Litten, v. Werra, Affanasiew, Gottschalk, Langhans beweisen; hier sehen wir analoge Verkalkungen auftreten in Fällen, wo von einer Entkalkung der Knochen gar nicht die Rede sein kann. Unterbindet man nach Litten die Arteria renalis eines Kaninchens 2 Stunden lang und löst dann die Ligatur, so sieht man in den nächsten Tagen die Epithelien, welche Coagulationsnekrose darbieten, sich mit Kalksalzen incrustiren. Anfangs sind die Epithelien noch erkennbar, vachher kommt es zu.Cylinderbildung aus confluirten Epithelien; in beiden Stadien sind die Kalkkrümel sichtbar. Leider geben die Bilder in der Litten: schen Arbeit die Verhältnisse nicht so gut wieder, dass man ohne Weiteres die Identität des Verkalkungsvorgangs bei der Sublimatniere erkennen kann. Macht man sich aber die kleine Mühe, experimentell eine solche Niere zu gewinnen, so wird man erstaunt sein über die Aehnlichkeit im mikroskopischen Befund beider Nieren, nur fanden wir nach der temporären Unterbindung der Nierenarterie die Kalksalze noch reichlicher eingelagert als in irgend einem Fall von tödtlich endender Sublimatintoxication mehrtägiger Dauer beim Kaninchen. Berücksichtigt man ferner, dass wir bei der Glycerin-, Aloin- und Wismuth-Niere gleichfalls Verkalkungen auf dem Boden der anämischen Epithelnekrose sehen, wie die oben genannten Forscher ausdrücklich betonen, so scheint es uns wohl berechtigt, jedenfalls die Annahme der Entkalkung der Knochen für überflüssig für das Zastandekommen der Kalkinfiltrationen bei der Sublimatniere zu halten. Uns scheint es auch nach der Prevost'schen Versuchsreihe keine so zwingende Nothwendigkeit zu sein, die acute Decalcinirung wirklich anzu- 
nehmen; dafür ist die Methode der Berechnung von Prevost nicht exact genug, ein Fehler, der, wie die Lectüre der Versuche lehrt, in der Natur der Sache liegt und keinen Vorwurf für den trefflichen Forscher involvirt.

Wenn wir aber selbst eine Decalcinirung der Knochen wirklich annehmen wollten, so würden wir doch in arge Verlegenheit kommen mit der Annahme einer oben nach Virchow definirten Kalkmetastase, denn unsere Präparate lassen keine andere Deutung zu, als dass der Kalk wesentlich in den Epithelien liegt, nicht im Lumen. Wenn ich diese Bahauptung so bündig der Virchow'schen Ansicht entgegen aufrecht halte, so thue ich dies sowohl auf Grund meiner eigenen Untersuchungen, als auch gestützt auf die Anschauungen der beiden ersten Untersucher und Entdecker der Kalkinfiltrationen bei der Sublimatniere, Saikowsky und Prevost. Mit diesen befinde ich mich in erfreulicher Harmonie. Es mögen daher meine beiden klassischen Zeugen zuerst für mich sprechen: Bei Saikowsky heist es zunächst:

„Die mikroskopische Untersuchung zeigte schon eine beträchtliche $A b$ lagerung im Epithel, welche bis zum Tode des Thieres an Volumen zunahm ${ }^{1}$."

Während dieser Passus unzweifelhaft für eine Incrustation der Epithelien spricht, könnte man in Betreff des folgenden vielleicht zweifelhaft sein, wohin Saikowsky den Kalk verlegt. Es heisst dort:

"Die in den Harnkanälchen abgelagerte Substanz stellt sich als amorphe, mehr oder weniger stark glänzende, stark das Licht reflectirende, gleichförmige Masse dar, welche das Lumen der Harnkanälchen so vollpfropft, dass man nicht im 'Stande ist, sein Epithel zu unterscheiden. Die Masse ist sowohl in kaltem, als anch in heissem sogar kochenden Alkohol und Aether unlöslich, löst sich aber sebr leicht in verdünnten Mineralsäuren (Schwefelsäure, $\mathrm{HCl}$ ), indem unter ziemlich unbedentender Gasentwicklung die dunkleu Massen versehwinden, das Feld des 0bjects klar wird, an den afficirten Stellen das Epithel wieder erseheint und eine mehr oder weniger bedentende Quantität von sternförmigen Krystallen sich bildet."

Prevost (Revue méd. de la Suisse romande. 1882), der den Vorgang der Veränderungen beim Menschen und bei seinen

1) Zur Erklärung sei bemerkt, dass Saik owsky Stäcke der Nieren der vergifteten Thiere successive exstirpirte und der Untersuchung unterwarf. 
Versuchsthieren identificirt, drückt sich in Betreff derselben also aus:

L'examen soigné des diverses phases de ce processus paraît indiquer que les cellules des tubuli droits, et plus tard celles des tubuli contorti subissent un certain dégré de tuméfaction, leur protoplasma et probablement modifié dans sa nature et ne tard pas à s'incruster de calcaire.

Und weiter:

On peut d'ailleurs, dans certains tubes observer l'envabissement progressif de cette calcification des cellules épithéliales qui offrant un aspect normal dans une partie du tube deviennent de plus en plus granuleuses et opaques.

Nach meinen Untersuchungen bin ich nun in Betreff des Modus der Ablagerung der Kalksalze zu dem gleichen Resultat gelangt. Fig. 1 stellt die Verhältnisse an der Kaninchenniere dar. Dort sieht man deutlich in fast normalem Gewebe gelegene grade Harnkanälchen, deren Epithelien zum Theil schlecht färbbar sind und eine schmutzig-graue Farbe annehmen, während andere desselben Kanälchens mit Hämatoxylin intensiv blangefärbt, also verkalkt sind '), wieder andere zeigen gute Kernfärbung und normales Aussehen.

Schon die ganze Anordnung lässt keine Zweifel darüber aufkommen, dass es sich um verkalkte Epithelien handelt; es fehlt jede Begrenzung durch ein Röhrenepithel und die Grösse stimmt genau mit derjenigen der nekrotischen, zur Verkalkung reifen Epithelien überein. Ohne Mühe lassen sich die schönsten Uebergänge constatiren. An dicken Schnitten wird eine Differenzirung natürlich schwer sein und man erhält den Eindruck einer Kalksäule, welche das Wandepithel einfach verdeckt; es ist deshalb geboten, recht dünne Schnitte zu machen, was nur durch eine gute Einbettung, am besten in Paraffin, gelingt.

1) Ieh benutzte das Hämatoxylin in der Form der Ziegler'scben Hämatoxylin-Alaun-Lösung, weil wir darin die feinste Farbenreaction a uf $\mathrm{Kalk}$ besitzen, wie wir aus den Untersuchungen von Strelzoff gelernt haben. (Dort färbt sich die Knorpelgrundsubstanz des wachsenden Knochens intensiv blau mit Hämatoxylin, wenn eine Ablagerung von Kalksalzen in dieselbe stattgefunden hat.) Es empfiehlt sich nur ganz burze Zeit die Farbe einwirken zu lassen, weil dann die Vorliebe der verkalkten Partien für dieselbe an schönsten hervortritt. 
Fixirt man die Schnitte dann mit Nelkenöl-Collodium und nimmt die Färbung auf dem Objectträger vor, so ist man sicher, Alles in seiner natürlichen Topographie erhalten zu haben. - Löst man den Kalk mit Säuren auf, so erscheinen die nekrotischen Zellen, mit welchen der Kalk sich verbunden hatte.

Zupft man die kleinen Kalkkrümel mit einer Nadel heraus, wie man das bei Trichinen macht, und schüttelt sie in einem Reagenzglas tüchtig mit Wasser, um etwa anhaftende Epithelien davon zu entfernen, so erhält man kleinste Krümelchen, nach deren Entkalkung meist deutlich eine organische Grundlage von der Form der nekrotischen Epithelien zurückbleibt. Da die nekrotischen Epithelien nun, wie wir oben sahen, öfters fast ganz mit einander verschmelzen und das Aussehen eines Cylinders erlangen können, so wird die Kalkincrustation zuweilen Producte liefern, welche ordinären Kalkinfarcten der graden Kanälchen täuschend ähnlich sehen, bei welchen sowohl Concretionen im Lumen der Kanälchen ${ }^{1}$ ) vorkommen können, als auch das Epithel an den betreffenden Stellen der Wand fehlen kann. Der Unterschied ist nur der, dass bei den gemeinen Kalkinfarcten grade die Tunicae propriae der gewöhnliche Sitz der Kalksalze sind, was bei der Sublimatniere nicht der Fall ist. Während ferner der gewöhnliche Kalkinfarct sich auch zuweilen in den Kapseln der Glomeruli findet, ist davon hier nicht die Rede. Wean in Fällen von Sublimatniere der Menschen Kalk an den Glomeruli beobachtet wurde, so stellt dies zweifellos einen ganz zufälligen, unwesentlichen Befund dar, der mit der Sublimatintoxication nichts zu thun hat ${ }^{2}$ ). Finden wir nun an einer Stelle einen klumpigen Cylinder, der von Epithelien gar nichts erkennen lässt und die ganze Masche des Harnkanälchelns vollgepfropft hat, so ist selbstverständig die Entscheidung, dass es sich hier um verkalkte, zusammengebackene Zellen handelt, nur

1) Auf das Vorkommen auch dieser Form bei der Sublimatniere und den Modus ibrer Entstehung werde ich nacher noch eingehen.

2) Fall bei Hallopeau, Du mercure action physiologique et thérapeupeutique, Paris 1878 und von Vircbow, Sitz. d. Berl. med. Gesellsch. 4. Jan. 1888. Der Befund würde ja auch direct gegen Virchow's Auffassung von der vollkommenen Aehnlichkeit der Verkalkung bei seiner. "Metastase". und der Sublimatniere sprechen, denn bei jener jst von einer Verkalkung der Glomeruli nirgendwo die Rede. 
möglich, wenn man sich durch Betrachtung vieler Präparate verschiedener Stadien mit den einzelnen Phasen dieses. Prozesses bekannt gemacht hat. Ich habe nun in den Figuren 3, 4, 5, 7, 8 Stadien abgebildet, welche das Verhältniss der Verkalkung illustriren. Auf Fig. 3 sieht man oben links bereits nekrotische, aber noch kalklose Epithelien, während dicht daneben die Epithelien mehr und mehr mit Kalk incrustirt sind. In Fig. 8 haben wir auf das Deutlichste in demselben gewundenen Harnkanälchen den Uebergang von der Nekrose obne Verkalkungen zu Epithelien, welche nekrotisch und mit Kalk beladen sind; während einzelne plumpe, vergrösserte, in den Kernen nicht tingirbare Epithelien nur erst wenige blaue Pünktchen zeigen, sind andere bereits ganz blau gefärbt. Fig. 7 zeigt ein gewundenes Harnkanälchen, dessen ganzer Epithelbesatz mehr oder weniger mit Kalk incrustirt ist. Im Lumen sehen wir feinkörnige Massen und verkalkte Schollen von der Gestalt der Epithelzellen und in engem Zusammenhang mit denselben. Ausserordentlich klar sind vor Allem Figuren 4, 5, 6. In Fig. 4 sehen wir den Epithelbesatz eines Kanälchens, der in 3 Zellen intensiv verkalkt ist, an einer Stelle beginnende Incrustation zeigt; in dem Lumen sehen wir einen hyalinen Cylinder. Dasselbe Verhältniss noch schöner zeigt Fig. 5 ; in den beiden nach oben gelegenen Abschuitten von Harnkanälchen sehen wir bereits geringe Kalkeinlagerung in den Epithelien, während in dem unteren ein Halbkreis von verkalkten Epithelien sich an einem hyalinen Cylinder anschmiegt. Fig. 6 ist ganz schwach mit Vesuvin gefärbt, wobei der Kalk nicht gefärbt erscheint ${ }^{1}$ ). Das Präparat entstammt meinem letzten Fall (Petzoldt), wo die Veränderungen besonders hochgradig waren und die topographischen Verhältnisse des Kalkes nicht so leicht zu bestimmen sind. In dem vorgeführten Bilde erleichtert der hyaline Cylinder, der

1) Färbt man stark mit Vesuvin, so färbt sich der Kalk sehr intensiv und wir erbalten genau die Bilder, wie sie Huber abbildet, jedoch als. nekrotische Epithelien bezeichnet. Es besteht daher für mich der leise Verdacht, dass es sich in den Huber'schen Präparaten doch vielleicht um Kalk handelt und es wäre gut gewesen, wenn Huber durch Angaben über das mikroskopische Verhalten dieser Stellen an ungefärbten Präparaten uns gleich über diesen Punkt beruhigt hätte, 
sich braun gefärbt hat, die Orientirung; das Lumen dieses Harnkanälchens muss der Grösse des Cylinders entsprechen, welcher schon durch seine Form als ächter Ausguss sich kennzeichnet. In der in Rede stehenden Niere, wo die hochgradigste Verkalkung herrscht, welche ich beobachtete, habe ich in ausserordentlich vielen Schnitten, in denen die Lagerung der Theile durch geeignete Methoden (Fixirung mit 50 pCt. Alkohol nach Biondi, und Aufkleben mit Nelkenöl-Collodium, letztes Verfahren noch viel schonender) garantirt war, kaum ein oder das andere Mal Kalk von Epithelien umgeben, also im Lumen, gesehen. Vielfach konnte ich mich aber selbst hier, wo wohl wegen der späten Vornahme der Section die Epithelien meist von der Membrana propria abgehoben waren, noch davon überzeugen, dass verkalkte Epithelien der Membrana propria aufsassen and auch an ihrer Form noch deutlich kenntlich waren ${ }^{1}$ ). Dass übrigens zuweilen einmal ein Kalkcylinder wirklich auch im Lumen liegen kann, das zu leugnen bin ich weit entfernt, und ich habe dieses Vorkommniss selbst, wenn auch sehr vereinzelt, bei meinen Nieren constatiren können. Jedenfalls ist das Vorkommen aber nicht die Regel und in den seltenen Fällen, wo ich es sah, so zu erklären, dass verkalkte und abgebröckelte Epithelien von höher gelegenen Abschnitten in tiefere fortgeschwemmt wurden. Schon Prevost erwähnt dieses Vorkommniss in folgendem Passus:

"Quelquefois cependant, quelques tubes de la substance médullaire contiennent des masses calcaires qui paraissent y avoir été retenues pendant la periode de leur élimination par les urines; mais on distingue alors que les épithélium qui les entourent sont restés sains, contrairement à ceux de la substance corticale qui sont gonflés, souvent incrustés; puis à un stade plus avancé, la calcification devenant plus forte, les épithélium ne sont plus distincts et la calcification des tubuli envahis offre l'apparence de masses compactes cassantes avec cassures brusques."

Fig. 9 zeigt uns eine Phase der Entwickelung des erwähnten Vorgangs und lässt über dessen Natur kaum einen Zweifel zu. An dem im Lumen liegenden Cylinder erkennen wir deatlich eine Zusammensetzung aus Epithelien, welche mit Kalk theilweise incrustirt sind.

1) Die Präparate habe ich seiner Zeit hier in der Schlesischen Gesellschaft fürr vaterländische Cultur vorzustellen Gelegenbeit gehạbt. 
Wir können demnach am Schlusse einer erneuerten Durchforschung einer sehr grossen Zahl von Präparaten unsere frühere Behauptung nur aufrecht erhalten, dass in der That eine Verkalkung vorher nekrotisch gewordener Epithelien bei der Sublimatniere statt hat nnd das Wesen der Kalkniere bedingt. Daraus folgt, dass es sich um eine „Kalkmetastase" hierbei schon aus rein histologischen Gründen nicht handeln kann, wenn anders, wie Virchow ${ }^{1}$ ) angiebt, bei derselben der Kalk im Lumen der Kanälchen liegt, die Concretionsbildung also hierbei genau übereinstimmt mit dem Modus, den Virchow - nach unserer Darstellung mit Unrecht für die Sublimat-Kalkniere als die Regel hinstellt. Aber selbst wenn bei der "Kalkmetastase" die Salze in den Epithelien; also in der Substanz der Nieren, abgelagert würden, wie das z. B. v. Recklinghausen ${ }^{2}$ ) anzunehmen scheint, so wäre es aus den oben entwickelten Gründen nicht nöthig, aus der dann bestehenden Aehnlichkeit mit unseren Sublimat-Kalknieren den Schluss zu ziehen, dass es sich darum auch bei dieser um eine Incrustation der Epithelien mit metastasirtem Knochenkalk handelt.

Von den erwähnten eigenthümlichen Kalkablagerungen in der Epithelschicht der Mucosa des Magens und Duodenums darf ich mit Rücksicht auf die zuerst von Virchow beschriebenen Kalkmetastasen in der Magenwand behaupten, dass sie mit denselben keinerlei Aehnlichkeit besitzen; im Uebrigen halte ich es nicht für gestattet, aus diesem bis jetzt vereinzelt dastehenden Befunde weitere Folgerungen zu ziehen. Dies würde vielleicht möglich sein, wenn diese Veränderungen noch in weiteren Fällen zur Beobachtung gelangen sollten.

\section{Erklärung der Abbildungen. Tafel VIII.}

Fig. 1. Kaninchenniere.

Fig. 2. Kalklose Sublimatniere

Fig. 3.) (Juni 1887).

Fig. 4. Sublimatkalkniere (Mai 1886). Fig. 5 .
Fig. 6. Sublimatkalkniere Petzoldt

Fig. 7.l

Fig. 8. Sublimatkalkniere (Mai 1886), Fig. 9.)

Erklärung im Text.

1) Berl. med. Gesellsch. Sitzg. vom 21. Nov. 1888 ,

2) Handbueh S, 174. 